\title{
Mechanism of miR-204-5p in exosomes derived from bronchoalveolar lavage fluid on the progression of pulmonary fibrosis via AP1S2
}

\author{
Liang Zhu ${ }^{1}$, Yahui Chen ${ }^{2}$, Mo Chen ${ }^{1}$, Wenwen Wang ${ }^{1}$ \\ ${ }^{1}$ Department of Rheumatism Immunology, the Second Affiliated Hospital, Zhejiang University School of Medicine, Hangzhou, China; ${ }^{2}$ Department \\ of Rheumatism Immunology, Ningbo Sixth Hospital, Ningbo, China \\ Contributions: (I) Conception and design: L Zhu; (II) Administrative support: L Zhu; M Chen; (III) Provision of study materials or patients: Y Chen; \\ W Wang; (IV) Collection and assembly of data: L Zhu; Y Chen; W Wang; (V) Data analysis and interpretation: L Zhu; Y Chen; M Chen; (VI) \\ Manuscript writing: All authors; (VII) Final approval of manuscript: All authors. \\ Correspondence to: Liang Zhu. Department of Rheumatism Immunology, the Second Affiliated Hospital, Zhejiang University School of Medicine, No. \\ 88, Jiefang Road, Shangcheng District, Hangzhou 310009, China. Email: zhuliang1059@zju.edu.cn.
}

Background: Exosomes are nanoscale vesicles secreted by various types of cells that are responsible for intracellular communication. Despite that bronchoalveolar lavage fluid (BALF) has been proven to involve in tumor development, more efforts are required to investigate the impact of BALF on pulmonary fibrosis (PF). This study aimed to investigate the mechanism of how exosomal miR-204-5p from BALF facilitates PF progression in rats.

Methods: PF rat model was established by intratracheal injection of bleomycin. BALF-derived exosomes (Exo) were extracted from normal and PF rats. PF-Exo (BALF-derived Exo from PF rats) and miR-204$5 \mathrm{p}$ antagomir were injected into rats to investigate the effect of exosomal miR-204-5p on PF. Collagen content in lung tissues of rats was assessed by Masson staining, hydroxyproline (HYP) content assay and immunohistochemistry (IHC). Primary lung fibroblasts were isolated, and treated by TGF- $\beta 1$. After cotransfection of PF-Exo, miR-204-5p inhibitor and sh-AP1S2, cell proliferation, levels of miR-204-5p, fibrotic markers $\alpha$-SMA and collagen 1 (Col 1), and proteins of autophagy markers LC3II, LC3I and P62 were measured. The interaction between miR-204-5p and AP1S2 was determined by bioinformatics online software TargetScan and dual-luciferase reporter assay.

Results: miR-204-5p was abundantly expressed in the PF-Exo group. PF-Exo injection potentiated PF progression and proliferation ability of lung fibroblasts in vivo and in vitro. Injection with PF-Exo and miR-204-5p antagomir significantly increased the LC3II/I ratio and decreased the HYP content, proteins of $\alpha$-SMA, Col 1 and P62, collagen content in rat lung tissues of PF rats. TGF- $\beta 1$ induction elevated the LC3II/LC3I ratio, suppressed the cell proliferation rate, and decreased the levels of $\alpha$-SMA, Col 1 and P62. Additionally, AP1S2 was a direct target of miR-204-5p. miR-204-5p inhibitor can counteract the effect of PF-Exo in proliferation of lung fibroblasts, while sh-AP1S2 eliminated the effect of miR-204-5p inhibitor.

Conclusions: Exosomal miR-204-5p from BALF inhibits autophagy to promote the progression of PF rats by targeting AP1S2.

Keywords: Bronchoalveolar lavage fluid-derived exosomes; miR-204-5p; AP1S2; autophagy; pulmonary fibrosis (PF)

Submitted Dec 17, 2020. Accepted for publication Apr 11, 2021.

doi: $10.21037 / \mathrm{atm}-20-8033$

View this article at: http://dx.doi.org/10.21037/atm-20-8033 


\section{Introduction}

Pulmonary fibrosis $(\mathrm{PF})$ is a chronic, progressive and irreversible interstitial pneumonia with high rates of mortality and mortality, and idiopathic PF (IPF) is the most severe form of PF (1-3). Moreover, IPF as one of the late outcomes of pulmonary diseases, is featured by destruction of the alveolar histoarchitecture, infiltration of immunocytes, and irregular changes in fibroblasts (4). IPF is associated with poor prognosis with a mean survival period of less than 5 years (5). Therefore, the development of efficient therapy for IPF is urgently needed.

Exosomes (Exo) are nanoscale vesicles ranging in size from $30 \mathrm{~nm}$ to $150 \mathrm{~nm}$, which originate from the endosomes by fusion of multivesicles with plasma membrane (6-9). Exo have been discovered in body fluids, including saliva, urine, breast milk, plasma, and the bronchoalveolar lavage fluid (BALF) (10-14). Growing evidence suggests that Exo play an essential role in various disease progression. For instance, Exo released by human umbilical cordmesenchymal stem cells can protect lipopolysaccharideinduced acute lung injury by inducing autophagy (15). Additionally, an inhalation treatment of secretome and Exo shows the therapeutic potential for lung regeneration in PF models (16). More recently, studies have demonstrated that BALF-derived Exo may cause inflammation by promoting cytokine secretion and leukotriene $\mathrm{C} 4$ production $(12,17)$. However, the effects of BALF-derived Exo on PF remain largely undetermined.

MicroRNA (miRNA) is a small non-coding RNA of approximately 22 nucleotides, which serves as posttranscriptional regulatory factor of its messenger RNA (mRNA) target by translational repression or mRNA degradation $(18,19)$. Interestingly, Exo can carry miRNA to affect recipient cell function, indicating that exosomal miRNAs play crucial role in intercellular communication (20-23). Former studies addressed that exosomal miRNAs could exert promotive effects on a variety of diseases in vitro. For example, exosomal miR-328 derived from M2 macrophages can facilitate PF progression (24). Exosomal miR-1247-3p induces cancer-associated fibroblast activation (25). Furthermore, exosomal miR-204-5p can efficiently suppress the proliferation of cancer cells by specifically regulating its target gene (26). As a frequently researched miRNA, miR-204-5p is responsible for biological behavior of cancer cells. In non-small cell lung cancer, miR-204-5p is implicated in the proliferation and autophagy of cancer cells (27). In chronic obstructive pulmonary disease, miR-204-5p was highly expressed in BLAF-derived exosomes (28). However, there is no information about the function of miR-204-5p or exosomal miR-204-5p in the pathogenesis of PF. AP1S2 is a protein that in humans is encoded by the AP1S2 gene. It encoded $\sigma 1 \mathrm{~B}$ subunit of the heterotetrameric adaptor protein-1 and found in the cytosolic side of coated vesicles in the Golgi compartment, which is responsible for the recognition of sorting signals of transmembrane receptors and the recruitment of clathrin (29). Existing study has reported that AP1S2 is a direct target of miR-204-5p (30). In light of recent data, we focused on exploring the potential interactions among exosomal miR-204-5p, AP1S2, and PF.

Herein, this research aimed to investigate the regulatory molecular mechanism of exosomal miR-204-5p from BALF in PF and its relationship with AP1S2. Our study elucidated that exosomal miR-204-5p derived from BALF may suppress autophagy to promote the progression of $\mathrm{PF}$ by targeting AP1S2. We present the following article in accordance with the ARRIVE reporting checklist (available at http://dx.doi.org/10.21037/atm-20-8033).

\section{Methods}

\section{Animals}

A total of 64 specific pathogen-free male Sprague Dawley (SD) rats (10-12 weeks old; weighing 220 \pm 20 g) were purchased from Beijing Vital River Lab Animal Technology Co., Ltd. (Beijing, China). Animal experiments were performed under a project license (No. 2020133) granted by the Ethics Committee of Zhejiang University, in compliance with the guidance of Animal Care and Use of Laboratory Animals.

\section{Establishment of PF rat models}

SD rats were acclimatized for 1 week at room temperature under free access to food and water. In this work, rats were subjected to intratracheal injection of bleomycin (Nippon Chemical Co., Ltd., Tokyo, Japan, $5 \mathrm{mg} / \mathrm{rat}$, dissolved in normal saline). The details for PF model establishment refer to information in a previous study (31). Briefly, rats were anesthetized with $1 \%$ pentobarbital sodium, and the skin covering the neck was dissected to expose the trachea. Then, $0.2 \mathrm{~mL}$ of bleomycin A5 $(5 \mathrm{mg} / \mathrm{kg})$ dissolved in normal saline was injected into the trachea of rats to construct PF rat models. 


\section{Isolation and characterization of Exo}

After tracheal intubation of normal rats $(\mathrm{n}=2)$ and $\mathrm{PF}$ rats $(\mathrm{n}=2)$, the lungs were gently lavaged with $1 \mathrm{~mL}$ of ice, sterile PBS for three times. The BALF collected from rats were stored at $-80^{\circ} \mathrm{C}$ and instantly processed for Exo isolation. Exo isolation was conducted by differential ultracentrifugation as showed in previous publications $(32,33)$. The protein concentration of Exo was utilized with the bicinchoninic acid (BCA) assay (Beyotime, Shanghai, China).

Transmission electron microscopy (TEM, Hitachi Limited, Japan) was employed to observe the morphology of Exo. A total of $20 \mu \mathrm{L}$ of Exo was added onto a copper mesh grid for $1 \mathrm{~min}$. Then, the Exo were dried with filter paper followed by adding 1 drop of $1 \%$ uranyl acetate before the Exo were dried again. After $1 \mathrm{~min}$, the Exo were visualized by TEM and imaged. Afterwards, Exo $(0.5 \mathrm{~mL})$ was diluted with ultrapure water $(4.5 \mathrm{~mL})$ and passed through a $0.22 \mu \mathrm{m}$ filter membrane, after which the size was determined by nanoparticle tracking analysis (NTA). Later, Western blot was conducted to assess the levels of the exosomal markers CD9, CD63 and Tsg101. Lastly, miR-204-5p expression was detected following the addition of RNase or RNase + Triton X-100 into culture medium to explore whether miR204-5 was expressed in the Exo. The Exo derived from normal and PF rats were renamed Sham-Exo and PF-Exo.

\section{Animal grouping}

All 60 rats were randomly divided into the following groups: Sham group (treated with $0.05 \mathrm{~mL}$ of PBS), PF group (PF model establishment followed by tail vein injection of normal saline), Sham-Exo group (tail vein injection of Exo derived from BALF of normal rats), PF-Exo group (tail vein injection of Exo derived from BALF of PF rats), PF-Exo + antagomir NC group (PF-Exo injection and antagomir NC treatment), and PF-Exo + miR-204-5p antagomir group (injection of PF-Exo and miR-204-5p antagomir). Twentyone days after model establishment, the rats were injected with Sham-Exo/PF-Exo $(0.5 \mathrm{mg} / \mathrm{kg} /$ day $)$ and equivalent normal saline separately, or simultaneously injected with PF-Exo $(0.5 \mathrm{mg} / \mathrm{kg} /$ day $)$ and antagomir NC or miR-204$5 \mathrm{p}$ antagomir $(10 \mathrm{mg} / \mathrm{kg}$ in $50 \mu \mathrm{L}$ saline), through the tail vein for consecutive 7 days. Antagomir NC and miR-204$5 \mathrm{p}$ antagomir were purchased from Shanghai GenePharma Co., Ltd. Company (Shanghai, China). On the $28^{\text {th }}$ day after modeling, lung tissues were collected. Histopathology was conducted by hematoxylin-eosin (H\&E) staining and Masson staining. Hydroxyproline (HYP) in lung tissues was detected using corresponding detection kit and the collected tissues were subjected to qRT-PCR and Western blot.

\section{Lung histomorphological analysis}

The lung tissues were fixed in $4 \%$ paraformaldehyde for $24 \mathrm{~h}$, and embedded in paraffin to prepare $4 \mu \mathrm{m}$ paraffin sections. Then, sections were subjected to $H \& E$ and Masson staining. The pathological changes of lung tissues were observed under an optical microscope, and 10 different fields were randomly selected for each slide. Ashcroft score was used to assess the severity of PF according to status of interstitial proliferation and integrity of alveolar structure (34). The score was recorded by two pathologists in double-blind trials and the average score was taken. Image-Pro Plus 6.0 software was adopted to calculate the ratio of Masson-stained blue area in the total area, and thus to evaluate the collagen deposition.

\section{Measurement of HYP content}

The biochemical detection of HYP reflects the collagen content in tissues. Approximately $100 \mathrm{mg}$ of lung tissues were homogenized. The experiment was hydrolyzed by alkali hydrolysis according to the manufacturer's instruction (Nanjing Jiancheng Biological Engineering Institute, Nanjing, China).

\section{Immunohistochemistry (IHC)}

The protein levels of $\alpha$-SMA and collagen 1 (Col 1) in lung tissues were detected by streptavidin peroxidase immunohistochemical kit. Paraffin sections were dewaxed by routine method, followed by $15 \mathrm{~min}$ of incubation with citrate antigen retrieval solution at $98{ }^{\circ} \mathrm{C}$. After rinsing with PBS, sections were blocked with goat serum and cultured with primary antibodies against $\alpha$-SMA (1:500, ab108424, Abcam, Cambridge, MA, USA) and Col 1 (1:200, ab34710, Abcam) at $4{ }^{\circ} \mathrm{C}$ before PBS rinsing. PBS was utilized as negative control of primary antibodies. Thereafter, sections were incubated with biotin-labeled second antibody at $37^{\circ} \mathrm{C}$ for $30 \mathrm{~min}$ and subsequently stained by DAB. Slides were counter-stained with hematoxylin, dehydrated, and mounted with neutral resin. Images were captured using an optical microscope. 


\section{Isolation and cultivation of cells}

Primary lung fibroblasts were isolated from SD rats. Briefly, rats were sacrificed after a single intratracheal injection of bleomycin sulfate $(5 \mathrm{mg} / \mathrm{mL})$ dissolved in sterile saline $(60 \mu \mathrm{L})$ for 7 days. The extracted lungs were rinsed 3 times with sterilized Hanks solution, after which the lung tissues were minced thoroughly and suspended in $0.5 \%$ trypsin $\left(10 \mathrm{~mL}, 37^{\circ} \mathrm{C}, 40 \mathrm{~min}\right)$. Afterwards, tissue suspension was centrifuged at 1,500 r/min for $5 \mathrm{~min}$ and the precipitates were suspended in Dulbecco's modified Eagle medium (DMEM), followed by centrifugation $(800 \mathrm{r} / \mathrm{min}, 5 \mathrm{~min})$. After another round of centrifugation (1,500 r/min, $5 \mathrm{~min}$ ), the precipitated cells were resuspended in $10 \mathrm{~mL}$ DMEM-F12 medium containing $1 \%$ antibiotics (streptomycin and penicillin) and $10 \%$ heat-inactivated fetal bovine serum (GIBCO, NY) in a humidified incubator at $37^{\circ} \mathrm{C}$ and $5 \% \mathrm{CO}_{2}$.

The cells were inoculated in $100 \mathrm{~mm}$ of culture dishes or 6-well plates for incubation after which the cells were randomly divided into the following 6 groups: Control group, TGF- $\beta_{1}$ group, PF-Exo group, PF-Exo + inhibitor $\mathrm{NC}+$ sh-NC group, PF-Exo + inhibitor NC + sh-AP1S2 group, and PF-Exo + miR-204-5p inhibitor + sh-AP1S2 group. TGF- $\beta_{1}(10 \mathrm{ng} / \mathrm{mL})$ was incubated in medium for $48 \mathrm{~h}$. Then, primary lung fibroblasts were incubated with $100 \mu \mathrm{g} / \mathrm{mL}$ of PF-Exo for $48 \mathrm{~h}$. Inhibitor NC, miR-204$5 \mathrm{p}$ inhibitor, sh-NC or sh-AP1S2 was then transfected into cells by using the Lipofectamine 2000 following the manufacturer's protocol. Thereafter, cells were collected for the following analysis. miR-204-5p inhibitor/inhibitor NC and sh-AP1S2/sh-NC were obtained from GenePharma (Shanghai, China).

\section{Internalization of Exo by pulmonary interstitial fibroblasts}

PKH67 dye (diluted at 1:1,000) was mixed with Exo suspension $(20 \mu \mathrm{g})$ and then incubated at $37^{\circ} \mathrm{C}$ for $15 \mathrm{~min}$ before the mixture was washed with PBS (70 min, 100,000 ×g). Afterwards, PKH67-labeled Exo and pulmonary interstitial fibroblasts were co-cultured for $30 \mathrm{~min}$. Finally, the uptake of Exo by pulmonary interstitial fibroblasts was observed by a confocal microscope.

\section{Acridine orange staining}

Primary lung fibroblasts $\left(5 \times 10^{4} / \mathrm{mL}\right)$ were inoculated onto 6-well plates. After that, the cells were treated with acridine orange solution at a final concentration of $1 \mathrm{mg} / \mathrm{L}$, which were cultured at $37{ }^{\circ} \mathrm{C}$ for $15 \mathrm{~min}$ and washed with PBS twice. Images were obtained by an inverted optical microscope (Nikon Ti-S, Nikon, Japan).

\section{EdU assay}

Cells in $5 \times 10^{3} /$ well were cultured with $100 \mu \mathrm{L}$ of $50 \mu \mathrm{M}$ EdU (Sigma-Aldrich, Merck KGaA, Darmstadt, Germany) at $37^{\circ} \mathrm{C}$ for $4 \mathrm{~h}$ before cells were fixed by $4 \%$ formaldehyde for $30 \mathrm{~min}$ and neutralized with glycine. Then, cells were cultured with reaction mixture of $1 \times$ Apollo $(100 \mu \mathrm{L}$, Ribbio, China) for 30 min before $0.5 \%$ Triton X-100 was used for permeation for $10 \mathrm{~min}$. Subsequently, cell nucleus was stained using Hoechst 33342 solution. Finally, cells were observed under a fluorescence microscope (Olympus, Tokyo, Japan).

\section{qRT-PCR}

Total RNAs were extracted from lung tissues and cells by utilizing TRIzol reagent. Then, RNA was reverse transcribed into cDNA using reverse transcription kit. The sequences of the primers for qRT-PCR synthesized by Sangon Biotech (Shanghai) Co., Ltd. (China) are indicated in Table 1. cDNA $(10 \mu \mathrm{L})$ was extracted and reactions were run on a Roche 480 real-time PCR instrument using SYBR ${ }^{\circledR}$ Green RealTime PCR Master Mix. The internal parameter of miR-204-5p was U6 and the internal parameter of AP1S2 was GAPDH. The data analysis was conducted by the $2^{-\Delta \Delta C t}$ method.

\section{Western blot analysis}

Lung tissues and cells of rats were lysed by RIPA containing proteinase inhibitor to extract proteins. Protein concentrations were measured with the BCA method (Beyotime, Shanghai, China), and then an appropriate amount of $5 \times$ SDS-PAGE sample buffer was added. Equal amount of protein $(15 \mu \mathrm{L})$ was electrophoresed with $10 \%$ SDS-PAGE at $300 \mathrm{~mA}$. The protein was then transferred onto a PVDF membrane and blocked with $5 \%$ skim milk powder. Subsequently, membranes were incubated with primary antibodies against LC3II/LC3I (Cell Signaling, \#12741, 1:1,000), P62 (Cell Signaling, \#88588, 1:1,000), and GAPDH (Cell Signaling Technology, Beverly, MA, USA, \#5174, 1:1,000) overnight at $4{ }^{\circ} \mathrm{C}$ before TBST washing, after which second antibody (Abcam, Cambridge, 
Table 1 Primer sequence for $\mathrm{qRT}$-PCR to determine the expression levels of miR-204-5p, AP1S2, Col 1, and $\alpha$-SMA

\begin{tabular}{ll}
\hline Name of primer & Sequences \\
\hline miR-204-5p-F & TCCGTATCCTACTGTT \\
miR-204-5p-R & GCAGGGTCCGAGGTATTC \\
U6-F & TCGCTTCGGCAGCACATATAC \\
U6-R & GCGTGTCATCCTTGCGCAG \\
AP1S2-F & CGAAGCTTCCCTGACGACT \\
AP1S2-R & TGTCAGGGCGTTTACTGTGC \\
COI 1-F & TGGGCATCTGCTTTAGCCTC \\
COI 1-R & TTCGATGACTGTCTTGCCCC \\
$\alpha-S M A-F$ & TGAAGGCGCTGATCCACAAA \\
$\alpha-S M A-R$ & AACCCTTGACACAAGGCCAA \\
GAPDH-F & ACCACAGTCCATGCCATCAC \\
GAPDH-R & TCCACCACCCTGTTGCTGTA \\
\hline
\end{tabular}

$F$, forward; $R$, reverse.

MA, USA, ab6728, 1:2,000) was added. After extensive washing with TBST, chemiluminescent solution was added. GAPDH was served as the internal control, and the results were analyzed by Image J software. LC3 is a marker of autophagy process and mainly involved in the formation of autophagosomes. LC3-II/LC3-I ratio is the hallmark of the degree of autophagy activation, and decreased ratio of LC3II/LC3I indicates suppressed autophagy level. P62, a classical receptor of autophagy, targets other proteins that bind to it for selective autophagy.

\section{Dual-luciferase reporter assay}

Bioinformatics online software TargetScan (http://www. targetscan.org/) was applied to predict the possible binding sites for miR-204-5p in the 3'UTR of the AP1S2 mRNA. Dual-luciferase reporter assay confirmed the interplay between miR-204-5p and AP1S2.

The AP1S2-3'UTR fragment containing the putative binding sequences of the wide-type AP1S2 (AP1S2-WT) and mutant AP1S2 (AP1S2-MUT) were cloned into pmirGLO vectors and these vectors were separately cotransfected with miR-204-5p mimic into primary lung fibroblasts. After $48 \mathrm{~h}$ of transfection, cells were collected. The activities of firefly luciferase and Renilla luciferase were measured using dual-luciferase assay (Beyotime, Shanghai, China) following the manufacturer's protocol.

\section{Statistical analysis}

SPSS 18.0 (IBM Corp., Armonk, NY, USA) and GraphPad Prism 7.0 (GraphPad Software Inc.) were conducted for statistical analyses. Data are represented as mean \pm standard deviation $\left(\bar{x}_{ \pm \mathrm{s}}\right)$. The $t$-test was used for comparison between two groups, and one-way ANOVA was used for comparison among multiple groups. $\mathrm{P}<0.05$ was deemed as statistically significance.

\section{Results}

\section{Isolation and identification of Exo}

Extensive evidences have demonstrated that Exo can be isolated from airway biofluids including BALF. To study the effects of BALF-derived Exo on PF rats, BALF-derived Exo were extracted from normal and PF rats and observed by TEM (Figure 1A). Besides, the particle size of Exo mainly distributed at $100 \mathrm{~nm}$ was probed by NTA (Figure $1 B$ ). Western blot determined the levels of exosomal markers CD9, CD63 and Tsg101 (Figure 1C), confirming the successful isolation of Exo.

\section{PF-Exo stimulates $P F$ in rats}

The Exo derived from normal or PF rats was injected into $\mathrm{PF}$ rats by tail vein after model establishment (Figure 2A). The morphology of lung tissues was evaluated by $\mathrm{H} \& \mathrm{E}$ staining. As shown in Figure 2B, the Sham group had normal structure of lung tissues with no obvious inflammatory cell infiltration. While the PF group and the Sham-Exo group appeared destroyed alveoli, thickened pulmonary interalveolar septa and infiltrated inflammatory cells. These effects were further aggravated in the PFExo group rather than in the PF group. Ashcroft score revealed that compared with the PF group, the Sham group had lower scores, whilst PF-Exo group had higher scores $(\mathrm{P}<0.05)$. There were no obvious changes between ShamExo group and $\mathrm{PF}$ group $(\mathrm{P}>0.05)$.

Next, collagen deposition in lung tissues in each group was measured by Masson staining, as shown in Figure 2C. Compared with the Sham group, the alveolar structures of $\mathrm{PF}$ and Sham-Exo groups were unclear and also showed a large amount of blue collagen deposition. In comparison with the PF group, these symptoms were further exacerbated in the PF-Exo group. HYP, one of the main components of collagen, reflects collagen content in lung tissues indirectly. As shown in Figure 2D, among Sham, 
A

Sham-Exo

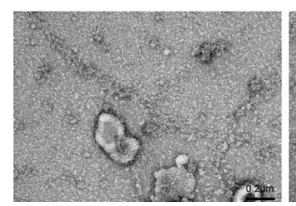

B

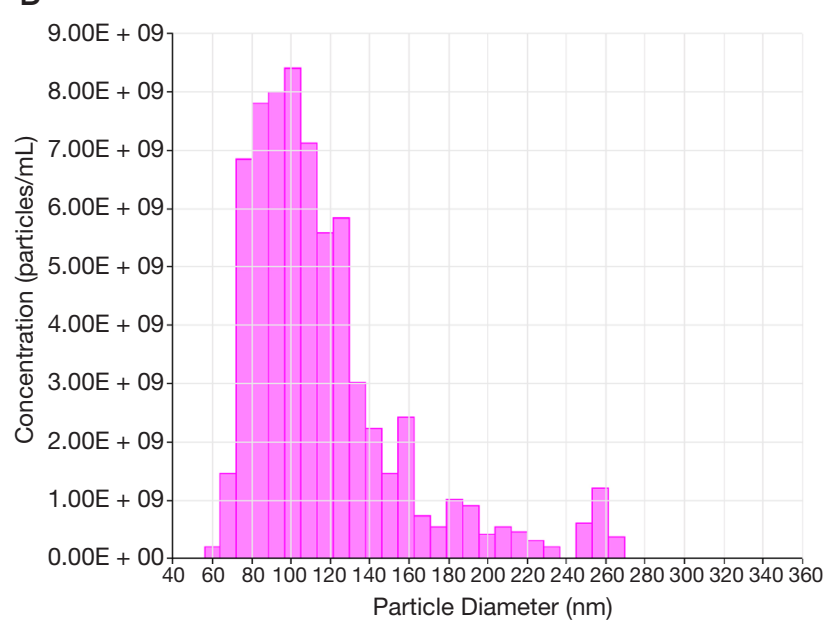

C
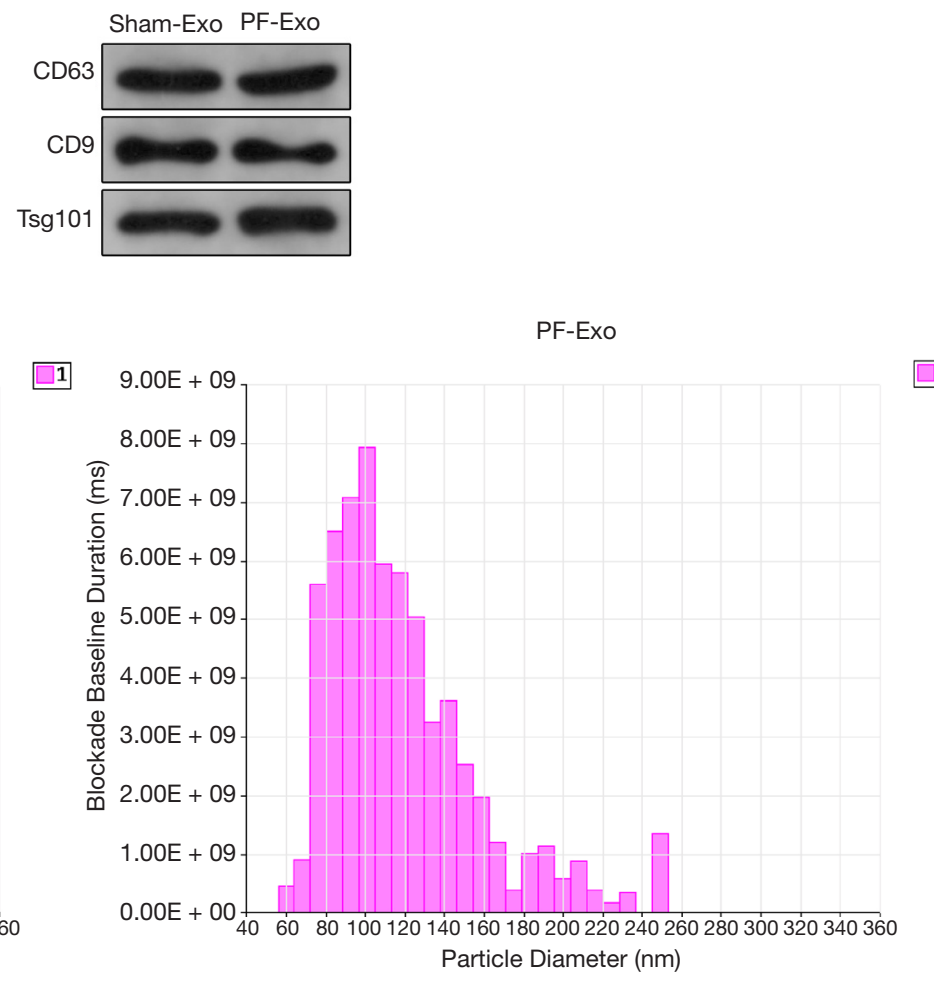

Figure 1 Successful isolation of Sham-Exo and PF-Exo. The morphology of BALF-derived Exo from normal and PF rats was analyzed using TEM (A). The particle size of Exo was assessed by NTA (B). Western blot analysis determined the levels of exosomal markers CD9, CD63 and Tsg101 (C). PF, pulmonary fibrosis; TEM, transmission electron microscopy; Exo, exosomes; NTA, nanoparticle tracking analysis.

PF and PF-Exo groups, the HYP content of the PF-Exo group was the highest, while the Sham group was the lowest $(\mathrm{P}<0.05)$. HYP content showed no significant changes between $\mathrm{PF}$ group and Sham-Exo group $(\mathrm{P}>0.05)$. These results indicated that the injection of PF-Exo can further promote the deposition of collagen fibers in the lung tissues of PF rats instead of the injection of Sham-Exo in rats.

To further determine the effect of BALF-derived Exo on $\mathrm{PF}$ rats, the protein expressions of fibrotic markers $\alpha$-SMA and Col 1 in lung tissues were assessed by IHC. The results displayed that in the Sham group, Col 1 was weakly positive and mainly distributed around bronchi, blood vessels and alveolar septa. In the PF group, Col 1 expression was significantly increased, especially in the bronchial and perivascular fibrosis area. In contrast to the PF group, the PF-Exo group had higher Col 1 expression (Figure 2E, $\mathrm{P}<0.05)$, while Col 1 expression remained unchanged in the Sham-Exo group (Figure $2 E, \mathrm{P}>0.05$ ). Besides, the Sham group showed that expression of $\alpha$-SMA was weakly positive and mainly detected in smooth muscle cells. However, expression of $\alpha$-SMA was significantly increased, and strong positive expression of $\alpha$-SMA was observed in the pulmonary interstitium, alveolar epithelium and peripheral vessel in the PF group. Compared with PF group, PF-Exo group had strongly $\alpha$-SMA expression (Figure $2 F, \mathrm{P}<0.05$ ), whilst there was no obvious change of $\alpha$-SMA expression between Sham-Exo and PF groups (Figure 2F, P>0.05).

\section{Downregulation of miR-204-5p mitigates PF-Exo induced $P F$ deterioration}

We found that injection of PF-Exo promoted the PF procession, while injection of Sham-Exo had no effects on PF rats. We further detected whether miR-204-5p was aberrantly expressed in PF-Exo. qRT-PCR described that miR-204-5p was highly expressed in the PF-Exo group (vs. Sham-Exo group) (Figure $3 A, \mathrm{P}<0.05$ ). Moreover, the expression of miR-204-5p remained unchanged after RNase treatment (vs. Control group), while miR-204-5p expression was decreased effectively after co-treatment of RNase 

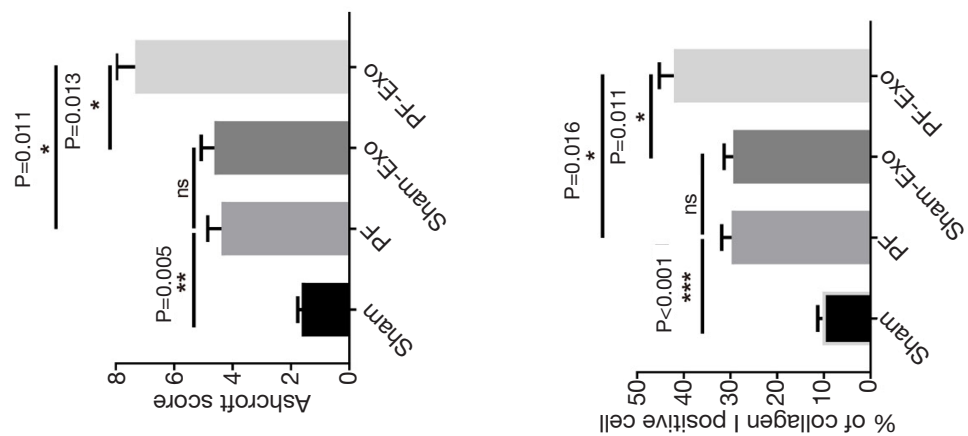

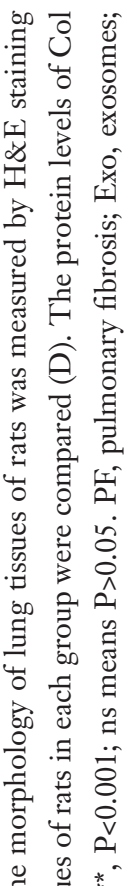

$\infty$
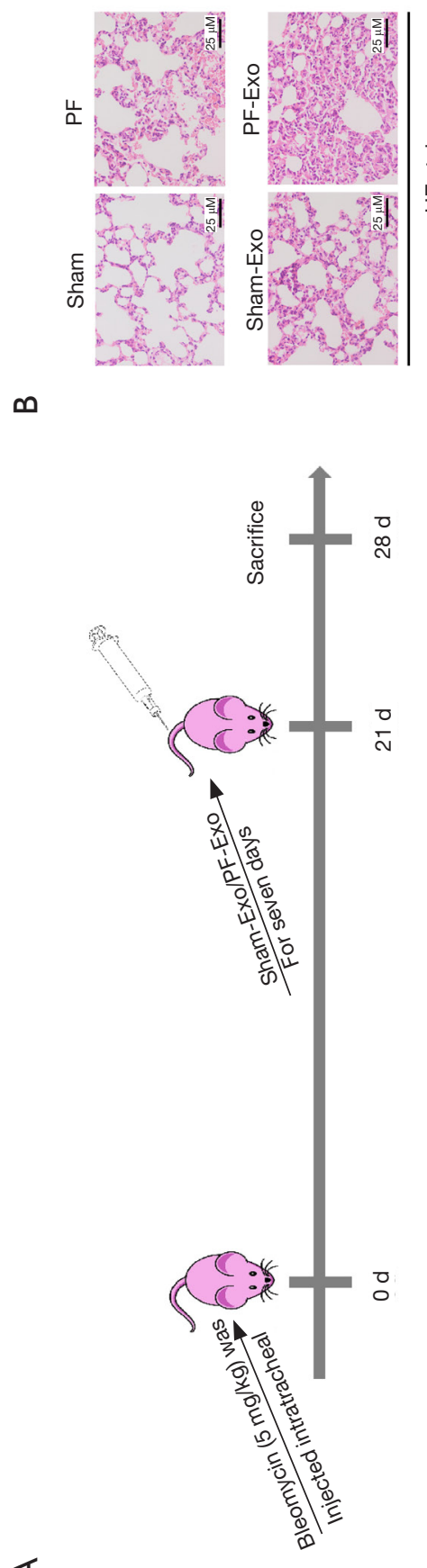

ш
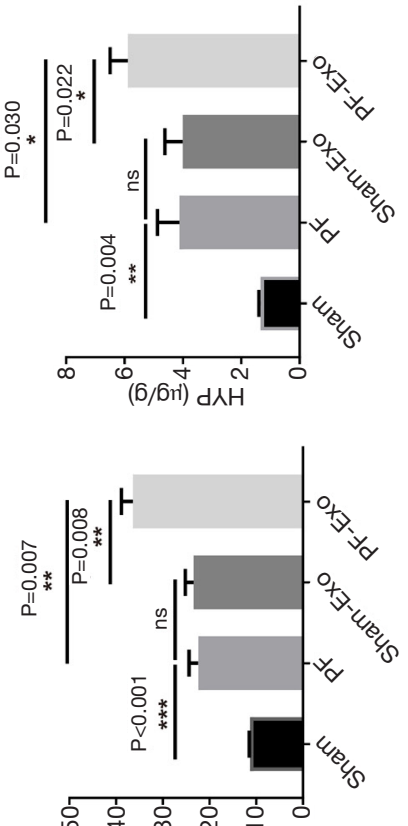

(\%) вәле иәбеп्०

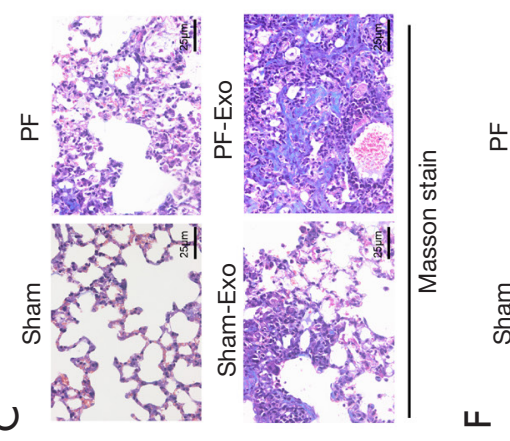

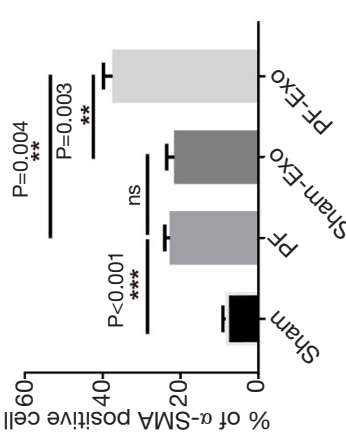

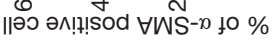
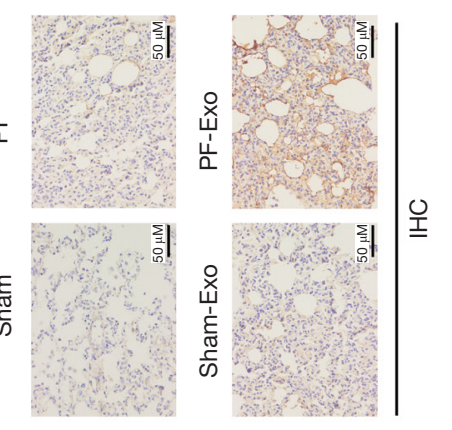

㝳

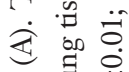

$\exists \Xi$

을

के

政

s)

氖 *

E

¿ิ

$\stackrel{0}{ \pm} \dot{1} \stackrel{D}{=}$

ठ․ㅀ

.

U

西

$\sum_{0}$

로 홀

苛. 己

ปี

U

य

我

$\exists$ ज

[

岂

స $\cong 0$

कั

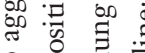

을

II

仙

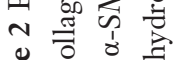

论 


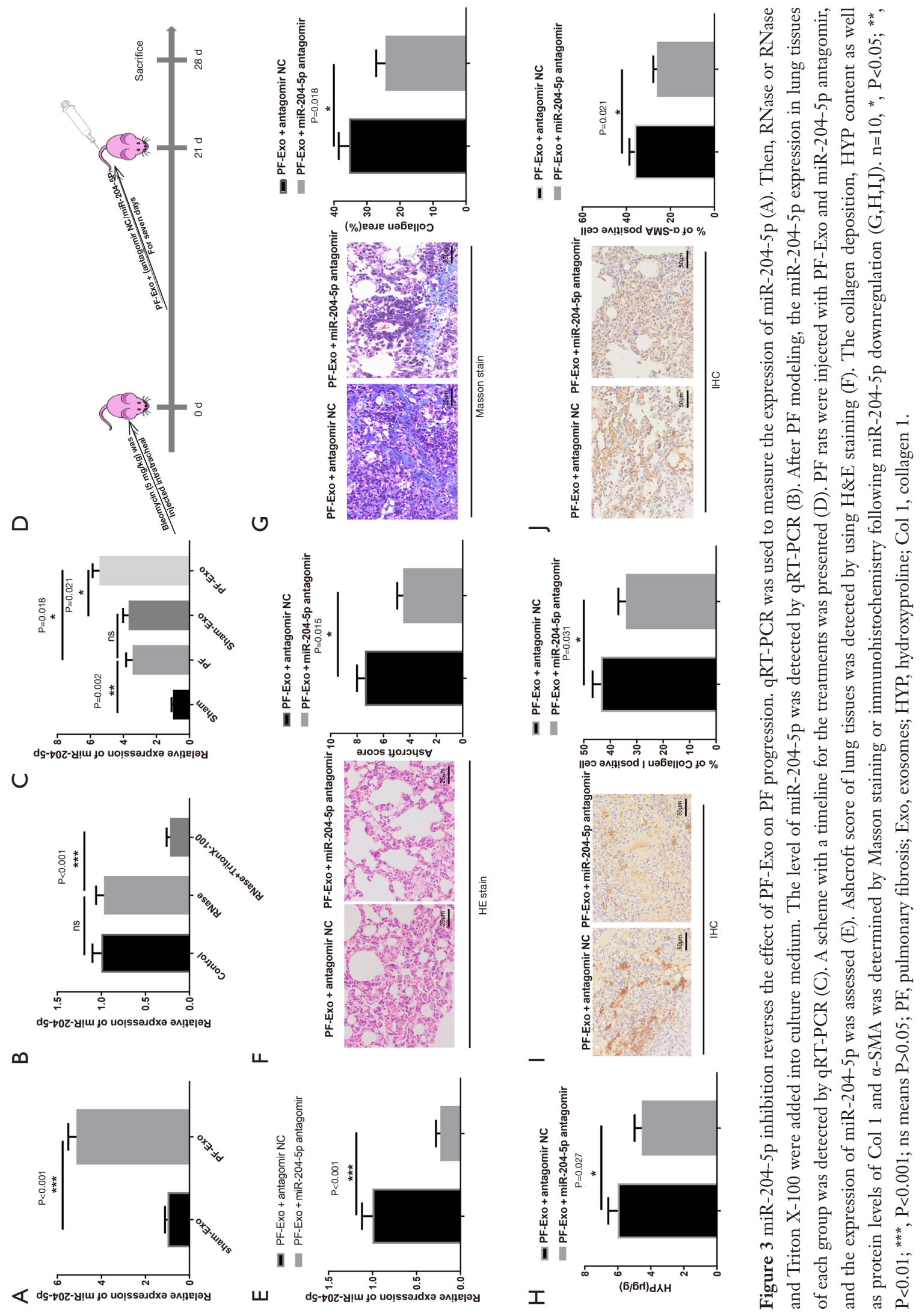


and TritonX-100 (Figure $3 B, \mathrm{P}<0.01$ ), which supported that miR-204-5p was membrane-enclosed. As shown in Figure $3 C$, the PF group had increased miR-204-5p expression (vs. Sham group), while PF-Exo group had elevated miR-204-5p expression (vs. $\mathrm{PF}$ group) $(\mathrm{P}<0.05)$. There was no significant change of miR-204-5p expression in Sham-Exo and PF groups $(\mathrm{P}>0.05)$. Above results supported that miR-204-5p may play an essential role in PF rats.

To determine the effect of exosomal miR-204-5p on $\mathrm{PF}$ rats, PF-Exo and miR-204-5p antagomir were injected into rats (Figure 3D). Lowly expressed miR-204-5p was noticed in the PF-Exo + miR-204-5p antagomir group rather than the PF-Exo + antagomir NC group (Figure 3E, $\mathrm{P}<0.01$ ). Additionally, $\mathrm{H} \& \mathrm{E}$ staining presented that in the PF-Exo + antagomir NC group, the alveolar structure was seriously damaged, and the surrounding alveolar septa were thickened with increased infiltration of the inflammatory cells, while these effects were alleviated in the PF-Exo + miR-204-5p antagomir group (Figure 3F). Ashcroft score displayed that the PF-Exo + miR-204-5p antagomir group had decreased scores (vs. PF-Exo + antagomir NC group) (Figure $3 G, \mathrm{P}<0.05$ ).

Next, collagen content in lung tissues in each group was analyzed by Masson staining, HYP content assay and IHC. The PF-Exo + antagomir NC group revealed a damaged alveolar structure and a large area of blue collagen deposition, however, such negative effects were ameliorated by injection of PF-Exo and miR-204-5p antagomir into rats. Besides, the decreases in collagen area, HYP content as well as protein levels of $\alpha$-SMA and Col 1 were found in the PF-Exo + miR-204-5p antagomir group (vs. PF-Exo + antagomir NC group) (Figure $3 H, I, 7, \mathrm{P}<0.05$ ). These data implied that downregulation of miR-204-5p could relieve $\mathrm{PF}$ procession promoted by PF-Exo.

\section{miR-204-5p from PF-Exo suppresses autophagy in PF rats}

External stimuli activate autophagy in lung tissues through eliminating pathogens, degrading damaged organelles and regulating inflammation, proliferation and apoptosis, thus exerting protective effects for lung tissues. The dysregulation of autophagy would accelerate the progression of lung diseases. Therefore, the levels of autophagy markers LC3II, LC3I and P62 in lung tissues of each group were analyzed by Western blot. The results showed that $\mathrm{PF}$ induction enhanced p62 level and diminished the ratio of LC3II/LC3I in lung tissues (the PF group $v s$. the Sham group), and these responses were further enhanced following PF-Exo injection $(\mathrm{P}<0.05)$, indicating PF-Exo inhibited autophagy. Compared with the PF-Exo + antagomir NC group, the PF-Exo + miR-204-5p antagomir group had increased ratio of LC3II/LC3I and decreased level of p62 in rat lung tissues (Figure 4, $\mathrm{P}<0.05$ ), suggesting that knockdown of miR-204-5p promoted autophagy. There was no obvious change in the LC3II/LC3I ratio and the expression of $\mathrm{p} 62$ between the $\mathrm{PF}$ and Sham-Exo groups $(\mathrm{P}>0.05)$. These results indicated that miR-204-5p from PF-Exo inhibited autophagy, and miR-204-5p knockdown could partially reverse the inhibitory effect of PF-Exo on autophagy in the lung tissues of PF rats. Combined with the above results, we concluded that PF-Exo may inhibit autophagy to promote $\mathrm{PF}$ by regulating exosomal miR-204-5p.

\section{PF-Exo inhibits autophagy and promotes proliferation of primary lung fibroblasts}

Primary lung fibroblasts were isolated from lung tissues to decipher the function of PF-Exo on PF. Firstly, primary lung fibroblasts were stimulated with TGF- $\beta_{1}$ and then incubated with Exo. The uptake of Exo by pulmonary interstitial fibroblasts was observed under confocal microscope, as shown in Figure 5A. Green fluorescence was observed in PKH67-labeled Exo. After 30 min of coculture of PKH67-labeled Exo and pulmonary interstitial fibroblasts, the slight green fluorescence of the PKH67labeled Exo was observed, suggesting that a small number of the PKH67-labeled Exo entered the pulmonary interstitial fibroblasts. Furthermore, with the extension of co-culture time, the pulmonary interstitial fibroblasts can absorb an increasing number of PKH67-labeled Exo. At 24 h, there was an obvious increase in the uptake of PKH67-labeled Exo, highlighting that pulmonary interstitial fibroblasts could internalize Exo. Besides, miR-204-5p was greatly increased in the TGF- $\beta_{1}$ group (vs. Control group), and further enhanced after co-culture with PF-Exo (Figure $5 B$, $\mathrm{P}<0.05$ ).

Uncontrolled cell proliferation of fibrosis-related cells is one of the causes of fibrosis. In this regards, the proliferation of primary lung fibroblasts was detected by EdU assay. The cell proliferation rate was increased in the TGF- $\beta_{1}$ group ( $v s$. Control group), and further elevated in the PF-Exo group (vs. TGF- $\beta_{1}$ group) (Figure $5 C, \mathrm{P}<0.05$ ).

Thereafter, the mRNA level of $\alpha$-SMA and Col 1 were measured by qRT-PCR. TGF- $\beta_{1}$ induction increased the 
mRNA levels of $\alpha$-SMA and Col 1 (the TGF- $\beta_{1}$ group $v s$. the Control group), which were further enhanced following co-treatment with PF-Exo (Figure 5D, $\mathrm{P}<0.05$ ). These data confirmed that PF-Exo may promote the proliferation of primary lung fibroblasts.

The effect of PF-Exo on autophagy was further verified. Acridine orange staining revealed that the number of acidic organelles was decreased in TGF- $\beta_{1}$-treated cells ( $v s$. Control group), and number of acidic organelles was further decreased after co-treatment with PF-Exo (vs. TGF- $\beta_{1}$ group) (Figure $5 \mathrm{E}, \mathrm{P}<0.05$ ).

Next, the protein levels of autophagy markers LC3II, LC3I and P62 were assessed by Western blot. TGF- $\beta_{1}$ group had decreased LC3II/LC3I ratio and increased P62 protein level (vs. Control group), suggesting that autophagy was inhibited in TGF- $\beta_{1}$-induced cells. LC3II/LC3I ratio was inhibited and $\mathrm{P} 62$ protein was increased in the PF-Exo group (vs. TGF- $\beta_{1}$ group) (Figure $5 F, \mathrm{P}<0.05$ ). Collective data corroborated that BALF-derived Exo inhibited autophagy and promoted proliferation of primary lung fibroblasts.

\section{AP1S2 is a target gene of miR-204-5p}

The target gene of miR-204-5p was predicted by TargetScan, and the results manifested that miR-204-5p specifically bind to the 3'UTR of AP1S2 mRNA to regulate AP1S2 expression, as depicted in Figure 6A. Further, dualluciferase reporter assay uncovered that the luciferase activity of AP1S2-WT in the miR-204-5p mimic group was strikingly dwindled (vs. mimic NC group) $(\mathrm{P}<0.01)$, but no notable difference of luciferase activity of AP1S2-MUT was found in the both miR-204-5p and mimic NC groups (Figure 6B, $\mathrm{P}>0.05$ ).

To further determine the targeting relationship between miR-204-5p and AP1S2, the mRNA and protein levels of AP1S2 were analyzed in the PF-Exo, Sham-Exo, mimic NC, miR-204-5p mimic, inhibitor NC and miR-204$5 \mathrm{p}$ inhibitor groups. The detection corroborated that the levels of AP1S2 were substantially decreased in the PFExo group (vs. Sham-Exo group) and miR-204-5p mimic group ( $v s$. mimic NC group), and prominently increased in the miR-204-5p inhibitor group (vs. inhibitor NC group) (Figure 6C,D, $\mathrm{P}<0.05$ ). Collectively, these findings clearly showed that overexpression of miR-204-5p could target and downregulate AP1S2 expression in the primary lung fibroblasts. 

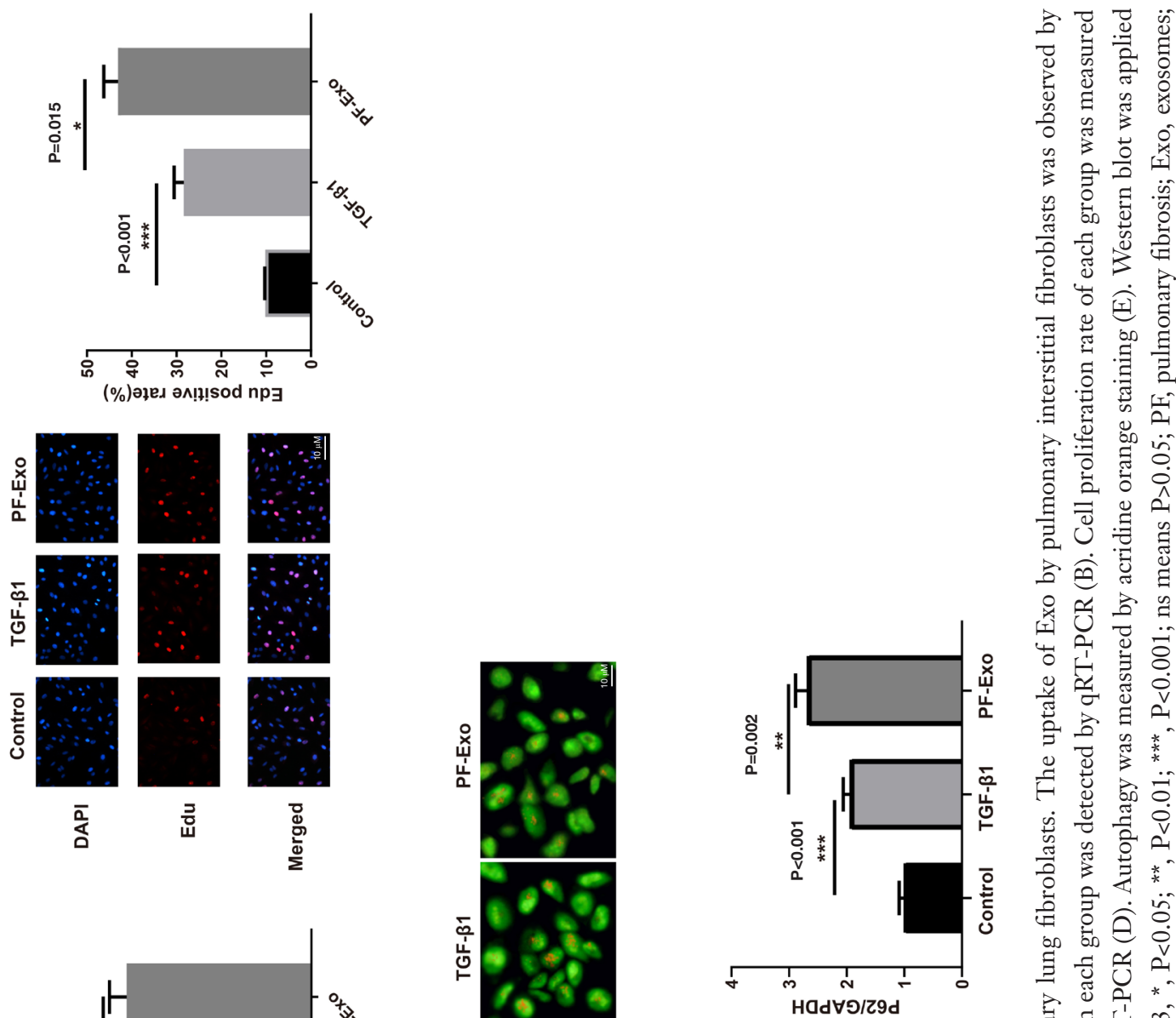

U

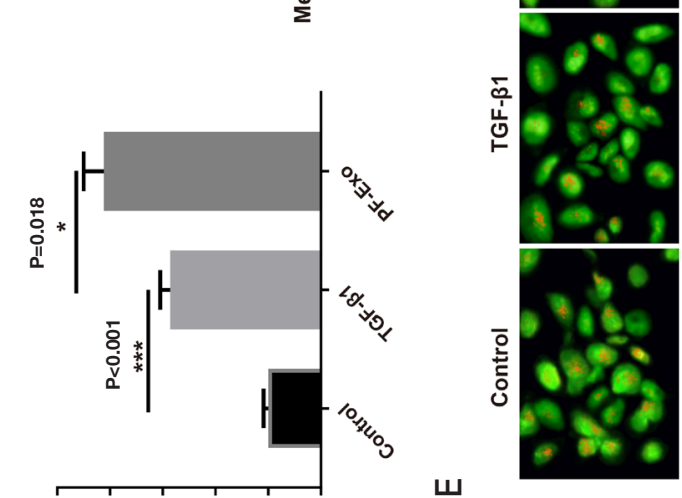

$\infty$

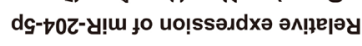
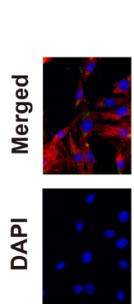

产

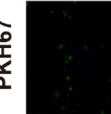

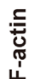

$\varangle$

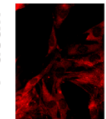

产
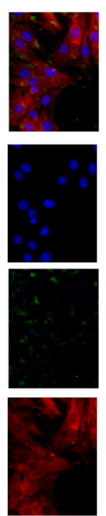

$\frac{\text { ล }}{\text { ล }}$
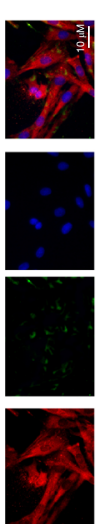

辛 ш

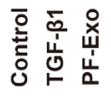

I

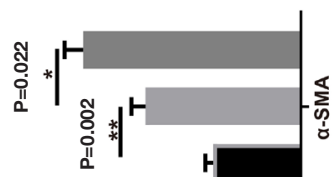

离 $* 1$

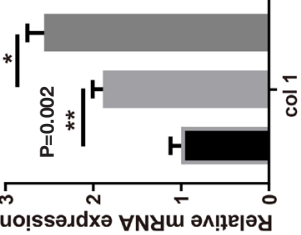

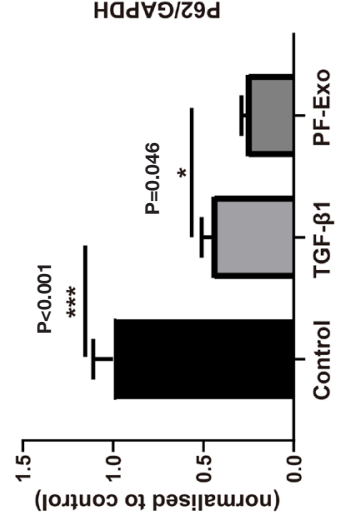

เยวา/|เยวา

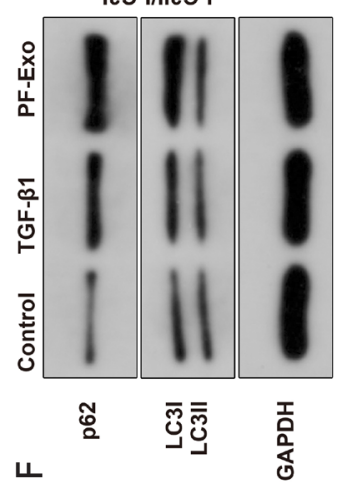

‥ 0 \%

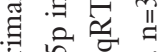

吉方全

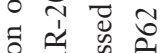

号当

यับ

च ळ

는

总 $\overline{0}$

过

论

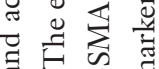

ส. 1

के

है.

笨

\& $\cong$ 范

仓े

ํㅡㄴㄹㅡ

के 开.

离 ठ

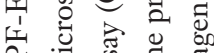

n

Ð

总 

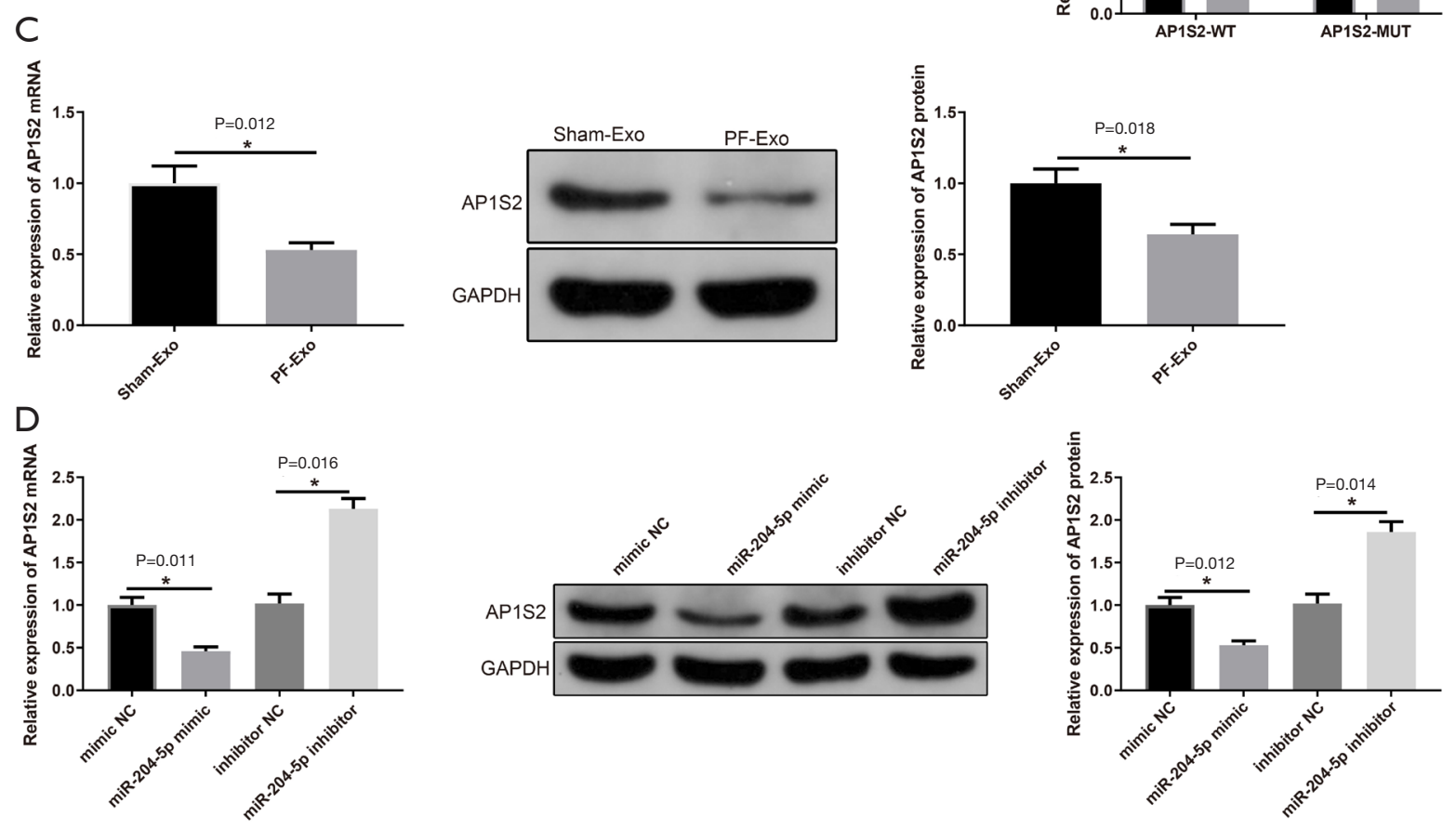

Figure 6 miR-204-5p negatively targets AP1S2. The binding sites between miR-204-5p and 3'UTR of AP1S2 were predicted using TargetScan (A). The interaction between AP1S2 and miR-204-5p was verified by dual-luciferase reporter assay (B). qRT-PCR and Western blot were used to assess the levels of AP1S2 in the PF-Exo and Sham-Exo group (C). The levels of AP1S2 in the mimic NC, miR-204-5p mimic, inhibitor NC and miR-204-5p inhibitor groups were determined by qRT-PCR and Western blot (D). n=3, * $\mathrm{P}<0.05 ;{ }^{* *}, \mathrm{P}<0.01 ; \mathrm{ns}$ means $\mathrm{P}>0.05$; PF, pulmonary fibrosis; Exo, exosomes.

\section{Exosomal miR-204-5p from BALF represses autophagy to promote lung fibroblast proliferation in vitro by regulating AP1S2 expression}

This step was further to ascertain the function of miR204-5p or AP1S2 on proliferation of lung fibroblasts. The detection on the levels of miR-204-5p and AP1S2 in the PF-Exo + inhibitor NC + sh-AP1S2 group and PFExo + miR-204-5p inhibitor + sh-AP1S2 group indicated high transfection efficiency (Figure $7 A, B$ ). Besides, cell proliferation was measured by EdU assay (Figure $7 C$ ) and the mRNA levels of $\alpha$-SMA and Col 1 were detected by qRT-PCR (Figure 7D). The detection showed that the PF-Exo + inhibitor NC + sh-AP1S2 group had increased proliferation rate in addition to elevated mRNA levels of $\alpha$-SMA and Col 1 (vs. PF-Exo + inhibitor NC + sh-NC group). However, the proliferation rate as well as mRNA levels of $\alpha$-SMA and Col 1 were prominently suppressed in the PF-Exo + miR-204-5p inhibitor + sh-AP1S2 group rather than the $\mathrm{PF}-\mathrm{Exo}+$ inhibitor $\mathrm{NC}+$ sh-AP1S2 group (Figure $7 C, D, \mathrm{P}<0.05$ ). These results implied that exosomal miR-204-5p from BALF may promote lung fibroblast proliferation through AP1S2 in vitro.

Acridine orange staining revealed that the PF-Exo + inhibitor NC + sh-AP1S2 group had decreased number of acidic organelles (vs. PF-Exo + inhibitor $\mathrm{NC}+$ sh-NC group), while PF-Exo + miR-204-5p inhibitor + sh-AP1S2 had elevated number of acidic organelles (vs. PF-Exo + inhibitor $\mathrm{NC}+$ sh-AP1S2 group) (Figure $7 E, \mathrm{P}<0.05$ ). The levels of autophagy-related proteins were determined by Western blot as shown in Figure $7 F$. The PF-Exo + inhibitor $\mathrm{NC}+$ sh-AP1S2 group had decreased LC3II/LC3I ratio 

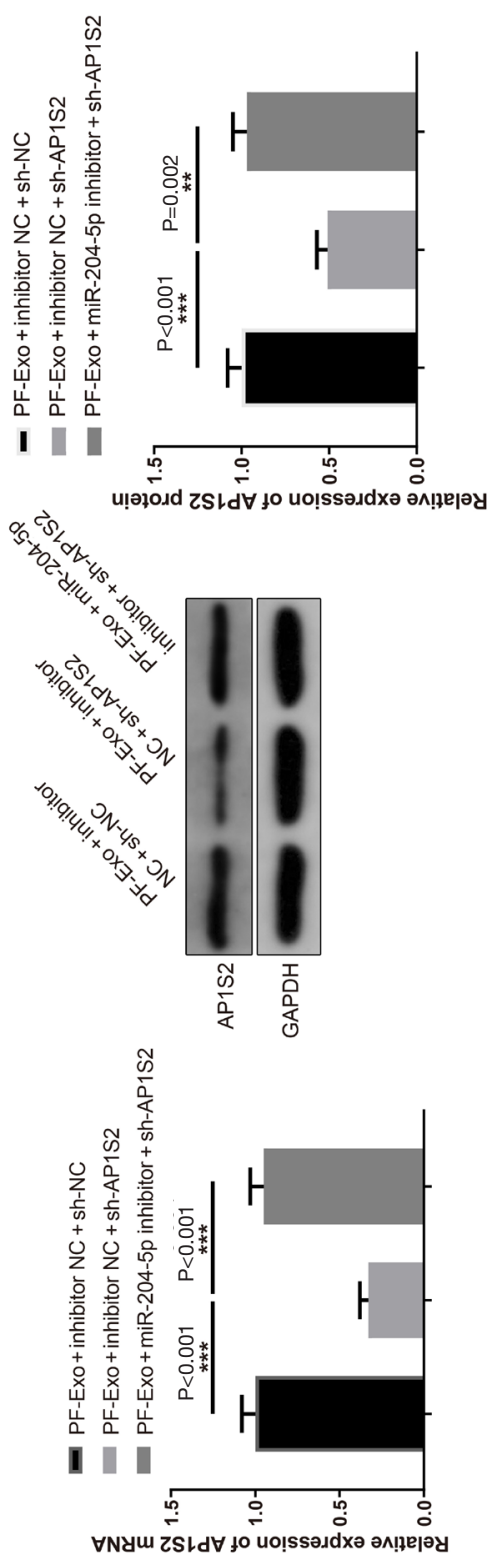

$\infty$

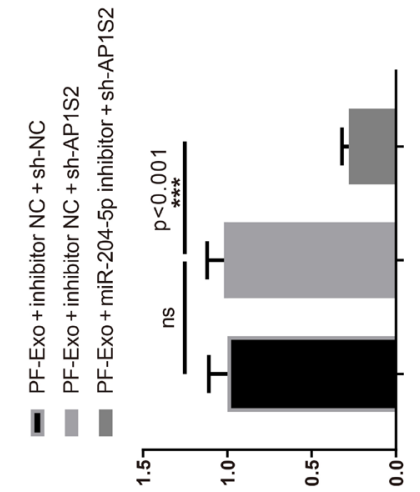

$\varangle$

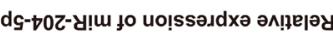

$\cup$
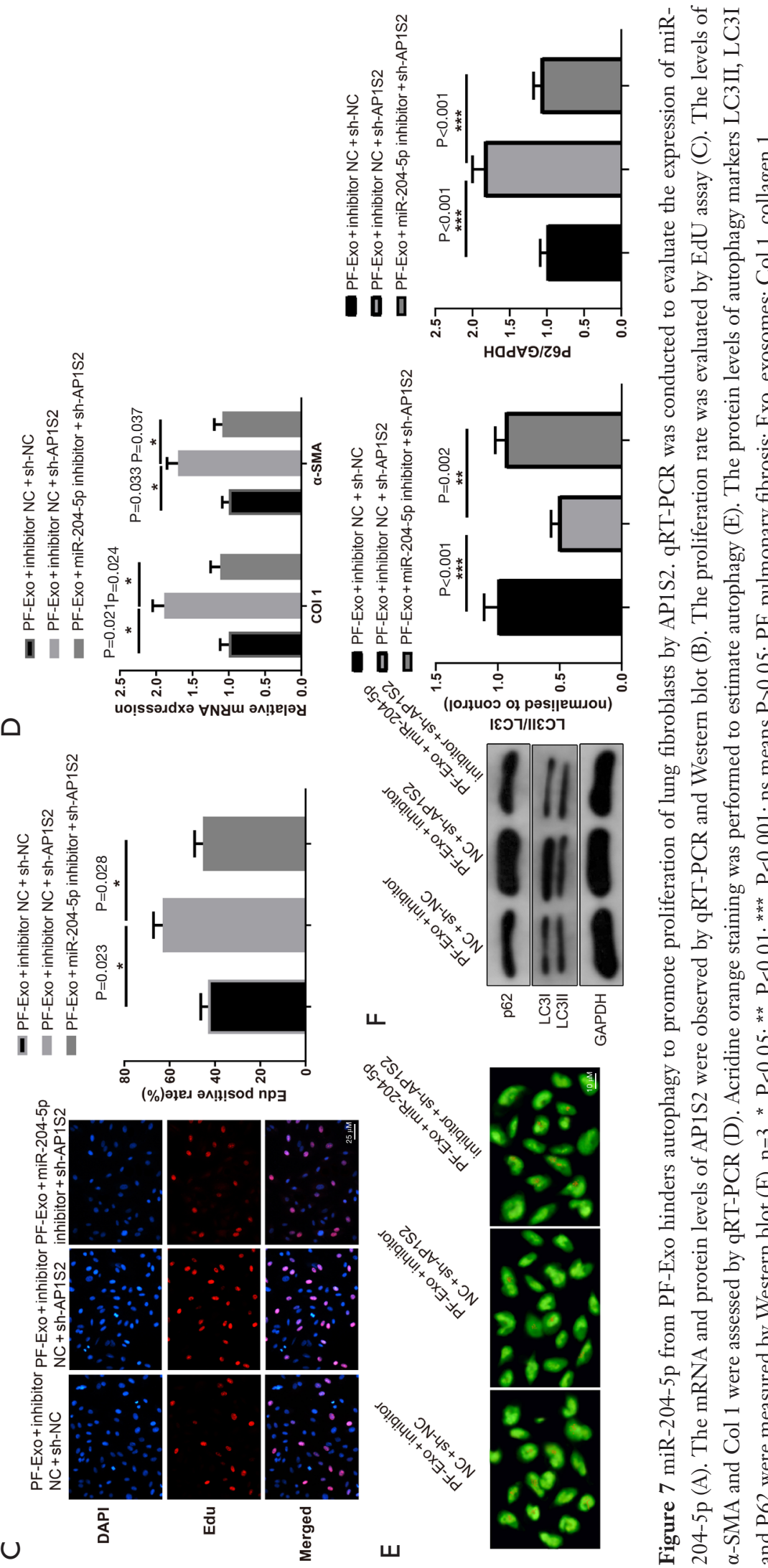

可

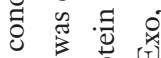

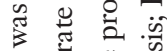

㚎.

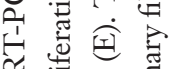

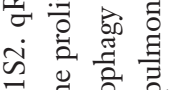

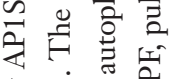

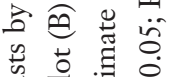

尊 $\frac{0}{0}$ 罗

苞荀

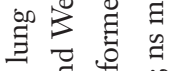

t)

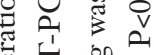

氙表贯

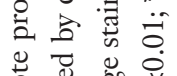

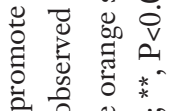

๖

कo

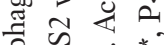

总完 $\hat{\Theta}^{*}$

के

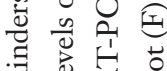

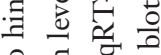

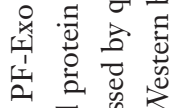

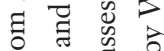

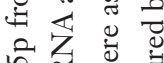

की

空 $\Rightarrow$

舟

定宊

o की

to 
and elevated P62 protein level (vs. PF-Exo + inhibitor NC + sh-NC group), while these effects were further enhanced in the PF-Exo + miR-204-5p inhibitor + sh-AP1S2 group ( $v$ s. $\mathrm{PF}-\mathrm{Exo}+$ inhibitor $\mathrm{NC}+$ sh-AP1S2 group $(\mathrm{P}<0.05)$. These results indicated that miR-204-5 $\mathrm{p}$ inhibitor promoted AP1S2 expression and activated autophagy. Taken together, miR-204-5p from PF-Exo inhibited autophagy to promote proliferation of lung fibroblasts by AP1S2 in vitro.

\section{Discussion}

In the present report, we sought to ascertain the effect of exosomal miR-204-5p from BALF on PF. Initially, we found high level of miR-204-5p in Exo derived from BALF of PF rats. Mechanistically, exosomal miR-204-5p from BALF inhibited autophagy and promoted the progression of PF by regulating AP1S2 expression. Collectively, the strategy focusing on exosomal miR-204-5p provided a novel therapeutic target for PF.

A growing volume of literature documented that Exo, as a specific type of extracellular vesicles existed in several biofluids including BALF could alter their composition in the process of diseases (35-37). Here, BALF-derived Exo were extracted from normal and PF rats in the present study. Previous study has revealed that BALF-derived Exo are an important indicator involved in lung cancer growth and progression (38). HYP is a key amino acid of collagen synthesis in fibrotic lung tissues (39). Our results found elevated HYP levels and obvious collagen deposition in lung tissues of rats after injection of PF-Exo. Thereafter, results from IHC disclosed that injection of PF-Exo had increased protein levels of fibrotic markers $\alpha$-SMA and Col 1 in lung tissues. Collectively, these findings corroborated that PFExo aggravates $\mathrm{PF}$ in rats.

Existing literature has unearthed abnormal expression of exosomal miRNAs in the progression of lung diseases, such as IPF, chronic obstructive pulmonary disease and asthma (28). For instance, exosomal miR-126, as a circulating biomarker, regulates cancer progression in nonsmall-cell lung cancer (40). Our report uncovered that BALF-derived Exo carrying miR-204-5p can enhance PF progression. Also, high level of miR-204-5p was observed during PF progression. Then, PF-Exo and miR-204-5p antagomir were injected into rats to further decipher the function of exosomal miR-204-5p on PF rats. We found that miR-204-5p expression was downregulated and the alveolar structure was improved with reduced alveolar septa and infiltration of the inflammatory cells. Also, there was an ameliorated alveolar structure and decreased blue collagen deposition. Besides, it also showed an increase of collagen, HYP content as well as protein levels of fibrotic markers. These data highlighted that overexpression of miR-204-5p could attenuate PF procession promoted by PF-Exo, albeit the mechanism is less well characterized.

A growing number of researches indicated that autophagy served a very important function on the development of human diseases $(41,42)$, including the progression of fibrotic disease (43). Besides, mounting evidences have reported that miR-204-5p could inhibit autophagy in various diseases including retinoblastoma, non-small cell lung cancer and diabetic retinopathy $(27,44,45)$. In the present study, the detection on the levels of autophagy markers LC3II, LC3I and P62 indicated that PF-Exo inhibited autophagy by carrying miR-204-5p in lung tissues, and miR-204-5p depletion could partially reverse the inhibition of PF-Exo on autophagy in the lung tissues of PF rats. One of the causes of fibrosis may due to the uncontrolled proliferation of fibrosis-related cells. TGF- $\beta$, as a growth factor, exerts a key role in fibroblast proliferation, affecting collagens and destroying lung structure to cause lung dysfunction $(46,47)$. A series of studies showed that cell proliferation rate as well as mRNA levels of fibrotic markers were significantly increased after TGF- $\beta_{1}$ treatment, which were further augmented following co-treatment of $\mathrm{PF}$ and Exo, indicating PF-Exo boost proliferation of primary lung fibroblasts. It was reported that TGF- $\beta 1$ stimulation suppressed autophagy flow in fibroblasts (48). Also, study has displayed that the decreased autophagy activity induced by TGF- $\beta 1$ facilitated the development of IPF (49). Consistently, our results presented that autophagy activity was significantly inhibited in lung fibroblasts treated with TGF- $\beta 1$, implicating BALF-derived Exo promoted proliferation of primary lung fibroblasts by inhibiting autophagy in vitro.

miRNAs are a type of non-coding RNA that could modulate the expression of target genes and a wide range of biological processes, including cell proliferation, apoptosis, differentiation, and metabolism (50). Given that exosomal miR-204-5p was up-regulated in PF, we tried to explore the downstream regulatory molecule of miR-204$5 \mathrm{p}$. Previously, the interaction between miR-204-5p and AP1S2 has been implicated in the metastasis of malignant melanoma (30). Here, our research proved that AP1S2 was a target gene a miR-204-5p and the levels of AP1S2 were substantially declined after transfection of PF-Exo or miR204-5p, indicating miR-204-5p could downregulate AP1S2 
expression in the primary lung fibroblasts. Besides, our findings suggested that miR-204-5p from PF-Exo promoted lung fibroblast proliferation through regulating AP1S2 expression in vitro. Also, the present study corroborated that miR-204-5p inhibitor promoted AP1S2 expression and activated autophagy. Together, these data clarified that miR-204-5p from PF-Exo inhibited autophagy to promote proliferation of lung fibroblasts in vitro by modulating AP1S2 expression.

This research clearly showed that exosomal miR-204-5p from BALF facilitated the progression of PF via inhibiting autophagy and AP1S2 in vitro and in vivo. However, these investigations had only been explored in vitro or in animals, and large amount of experiments should be conducted before these treatments yield health benefits in the clinic. Although miR-based therapeutics are still in the infancy, our study found that miR-204-5p can be regarded as a key element in the course of $\mathrm{PF}$ and as potent therapeutic target in the future.

\section{Acknowledgments}

Thanks for all the contributors and participants.

Funding: This research was supported by the grants from Medical Health Science and Technology Planning Project of Zhejiang Provincial Health Commission (Grant No. 2017192934) and Science and Technology Planning Project of Zhejiang Province (Grant No. 2017C37128).

\section{Footnote}

Reporting Checklist: The authors have completed the ARRIVE reporting checklist. Available at http://dx.doi. org/10.21037/atm-20-8033

Data Sharing Statement: The datasets used or analyzed during the current study are available from the corresponding author on reasonable request. Available at http://dx.doi.org/10.21037/atm-20-8033

Conflicts of Interest: All authors have completed the ICMJE uniform disclosure form (available at http://dx.doi. org/10.21037/atm-20-8033). The authors have no conflicts of interest to declare.

Ethical Statement: The authors are accountable for all aspects of the work in ensuring that questions related to the accuracy or integrity of any part of the work are appropriately investigated and resolved. Animal experiments were performed under a project license (No. 2020133) granted by the Ethics Committee of Zhejiang University, in compliance with the guidance of Animal Care and Use of Laboratory Animals.

Open Access Statement: This is an Open Access article distributed in accordance with the Creative Commons Attribution-NonCommercial-NoDerivs 4.0 International License (CC BY-NC-ND 4.0), which permits the noncommercial replication and distribution of the article with the strict proviso that no changes or edits are made and the original work is properly cited (including links to both the formal publication through the relevant DOI and the license). See: https://creativecommons.org/licenses/by-nc-nd/4.0/.

\section{References}

1. Li LC, Kan LD. Traditional Chinese medicine for pulmonary fibrosis therapy: Progress and future prospects. J Ethnopharmacol 2017;198:45-63.

2. Meyer KC. Pulmonary fibrosis, part I: epidemiology, pathogenesis, and diagnosis. Expert Rev Respir Med 2017;11:343-59.

3. Wynn TA. Integrating mechanisms of pulmonary fibrosis. J Exp Med 2011;208:1339-50.

4. Martinez FJ, Collard HR, Pardo A, et al. Idiopathic pulmonary fibrosis. Nat Rev Dis Primers 2017;3:17074.

5. Bargagli E, Di Masi M, Perruzza $M$, et al. The pathogenetic mechanisms of cough in idiopathic pulmonary fibrosis. Intern Emerg Med 2019;14:39-43.

6. Colombo M, Raposo G, Thery C. Biogenesis, secretion, and intercellular interactions of exosomes and other extracellular vesicles. Annu Rev Cell Dev Biol 2014;30:255-89.

7. Théry C, Ostrowski M, Segura E. Membrane vesicles as conveyors of immune responses. Nat Rev Immunol 2009;9:581-93.

8. Théry C, Zitvogel L, Amigorena S. Exosomes: composition, biogenesis and function. Nat Rev Immunol 2002;2:569-79.

9. Witwer KW, Buzas EI, Bemis LT, et al. Standardization of sample collection, isolation and analysis methods in extracellular vesicle research. J Extracell Vesicles 2013. doi: 10.3402/jev.v2i0.20360.

10. Katsiougiannis S, Chia D, Kim Y, et al. Saliva exosomes from pancreatic tumor-bearing mice modulate NK cell phenotype and antitumor cytotoxicity. FASEB J 
2017;31:998-1010.

11. Rodríguez M, Bajo-Santos C, Hessvik NP, et al. Identification of non-invasive miRNAs biomarkers for prostate cancer by deep sequencing analysis of urinary exosomes. Mol Cancer 2017;16:156.

12. Torregrosa Paredes P, Esser J, Admyre C, et al. Bronchoalveolar lavage fluid exosomes contribute to cytokine and leukotriene production in allergic asthma. Allergy 2012;67:911-9.

13. Torregrosa Paredes P, Gutzeit C, Johansson S, et al. Differences in exosome populations in human breast milk in relation to allergic sensitization and lifestyle. Allergy 2014;69:463-71.

14. Vicencio JM, Yellon DM, Sivaraman V, et al. Plasma exosomes protect the myocardium from ischemiareperfusion injury. J Am Coll Cardiol 2015;65:1525-36.

15. Wei X, Yi X, Lv H, et al. MicroRNA-377-3p released by mesenchymal stem cell exosomes ameliorates lipopolysaccharide-induced acute lung injury by targeting RPTOR to induce autophagy. Cell Death Dis 2020;11:657.

16. Dinh PC, Paudel D, Brochu H, et al. Inhalation of lung spheroid cell secretome and exosomes promotes lung repair in pulmonary fibrosis. Nat Commun 2020;11:1064.

17. Qazi KR, Torregrosa Paredes P, Dahlberg B, et al. Proinflammatory exosomes in bronchoalveolar lavage fluid of patients with sarcoidosis. Thorax 2010;65:1016-24.

18. Catalanotto C, Cogoni C, Zardo G. MicroRNA in Control of Gene Expression: An Overview of Nuclear Functions. Int J Mol Sci 2016;17:1712.

19. Xu J, Pan X, Hu Z. MiR-502 mediates esophageal cancer cell TE1 proliferation by promoting AKT phosphorylation. Biochem Biophys Res Commun 2018;501:119-23.

20. Li FX, Liu JJ, Xu F, et al. Role of tumor-derived exosomes in bone metastasis. Oncol Lett 2019;18:3935-45.

21. Valadi H, Ekstrom K, Bossios A, et al. Exosome-mediated transfer of mRNAs and microRNAs is a novel mechanism of genetic exchange between cells. Nat Cell Biol 2007;9:654-9.

22. Wang J, Deng Z, Wang Z, et al. MicroRNA-155 in exosomes secreted from helicobacter pylori infection macrophages immunomodulates inflammatory response. Am J Transl Res 2016;8:3700-9.

23. Yin W, Ouyang S, Luo Z, et al. Immature Exosomes Derived from MicroRNA-146a Overexpressing Dendritic Cells Act as Antigen-Specific Therapy for Myasthenia Gravis. Inflammation 2017;40:1460-73.

24. Yao MY, Zhang WH, Ma WT, et al. microRNA-328 in exosomes derived from M2 macrophages exerts a promotive effect on the progression of pulmonary fibrosis via FAM13A in a rat model. Exp Mol Med 2019;51:1-16.

25. Fang T, Lv H, Lv G, et al. Tumor-derived exosomal miR1247-3p induces cancer-associated fibroblast activation to foster lung metastasis of liver cancer. Nat Commun 2018;9:191.

26. Yao S, Yin Y, Jin G, et al. Exosome-mediated delivery of miR-204-5p inhibits tumor growth and chemoresistance. Cancer Med 2020;9:5989-98.

27. Kang Y, Jia Y, Wang Q, et al. Long Noncoding RNA KCNQ1OT1 Promotes the Progression of Non-Small Cell Lung Cancer via Regulating miR-204-5p/ATG3 Axis. Onco Targets Ther 2019;12:10787-97.

28. Guiot J, Struman I, Louis E, et al. Exosomal miRNAs in Lung Diseases: From Biologic Function to Therapeutic Targets. J Clin Med 2019;8:1345.

29. Huo L, Teng Z, Wang H, et al. A novel splice site mutation in AP1S2 gene for X-linked mental retardation in a Chinese pedigree and literature review. Brain Behav 2019;9:e01221.

30. Luan W, Ding Y, Ma S, et al. Long noncoding RNA LINC00518 acts as a competing endogenous RNA to promote the metastasis of malignant melanoma via miR204-5p/AP1S2 axis. Cell Death Dis 2019;10:855.

31. Maeyama T, Kuwano K, Kawasaki M, et al. Attenuation of bleomycin-induced pneumopathy in mice by monoclonal antibody to interleukin-12. Am J Physiol Lung Cell Mol Physiol 2001;280:L1128-37.

32. Murphy TF, Brauer AL, Sethi S, et al. Haemophilus haemolyticus: a human respiratory tract commensal to be distinguished from Haemophilus influenzae. J Infect Dis 2007;195:81-9.

33. Prado N, Marazuela EG, Segura E, et al. Exosomes from bronchoalveolar fluid of tolerized mice prevent allergic reaction. J Immunol 2008;181:1519-25.

34. Ashcroft T, Simpson JM, Timbrell V. Simple method of estimating severity of pulmonary fibrosis on a numerical scale. J Clin Pathol 1988;41:467-70.

35. Admyre C, Grunewald J, Thyberg J, et al. Exosomes with major histocompatibility complex class II and costimulatory molecules are present in human BAL fluid. Eur Respir J 2003;22:578-83.

36. Gallo A, Tandon M, Alevizos I, et al. The majority of microRNAs detectable in serum and saliva is concentrated in exosomes. PLoS One 2012;7:e30679.

37. Njock MS, Fish JE. Endothelial miRNAs as Cellular Messengers in Cardiometabolic Diseases. Trends 
Endocrinol Metab 2017;28:237-46.

38. Yang Y, Ji P, Wang X, et al. Bronchoalveolar Lavage FluidDerived Exosomes: A Novel Role Contributing to Lung Cancer Growth. Front Oncol 2019;9:197.

39. Yang X, Wu L, Li G, et al. Alphacalcidol combined with dexamethasone for reducing pulmonary fibrosis in mice and its mechanism. Xi Bao Yu Fen Zi Mian Yi Xue Za Zhi 2017;33:488-91.

40. Grimolizzi F, Monaco F, Leoni F, et al. Exosomal miR-126 as a circulating biomarker in non-small-cell lung cancer regulating cancer progression. Sci Rep 2017;7:15277.

41. Guo F, Liu X, Cai H, et al. Autophagy in neurodegenerative diseases: pathogenesis and therapy. Brain Pathol 2018;28:3-13.

42. Yu S, Wang Y, Jing L, et al. Autophagy in the "inflammation-carcinogenesis" pathway of liver and HCC immunotherapy. Cancer Lett 2017;411:82-9.

43. Han R, Ji X, Rong R, et al. MiR-449a regulates autophagy to inhibit silica-induced pulmonary fibrosis through targeting Bcl2. J Mol Med (Berl) 2016;94:1267-79.

44. Mao XB, Cheng YH, Xu YY. miR-204-5p promotes diabetic retinopathy development via downregulation of microtubule-associated protein 1 light chain 3. Exp Ther

Cite this article as: Zhu L, Chen Y, Chen M, Wang W. Mechanism of miR-204-5p in exosomes derived from bronchoalveolar lavage fluid on the progression of pulmonary fibrosis via AP1S2. Ann Transl Med 2021;9(13):1068. doi: 10.21037/atm-20-8033
Med 2019;17:2945-52.

45. Yao L, Yang L, Song H, et al. Silencing of lncRNA XIST suppresses proliferation and autophagy and enhances vincristine sensitivity in retinoblastoma cells by sponging miR-204-5p. Eur Rev Med Pharmacol Sci 2020;24:3526-37.

46. Kyung SY, Kim DY, Yoon JY, et al. Sulforaphane attenuates pulmonary fibrosis by inhibiting the epithelialmesenchymal transition. BMC Pharmacol Toxicol 2018;19:13.

47. Sibinska Z, Tian X, Korfei M, et al. Amplified canonical transforming growth factor- $\beta$ signalling via heat shock protein 90 in pulmonary fibrosis. Eur Respir J 2017;49:1501941.

48. Sosulski ML, Gongora R, Danchuk S, et al. Deregulation of selective autophagy during aging and pulmonary fibrosis: the role of TGFbeta1. Aging Cell 2015;14:774-83.

49. Patel AS, Lin L, Geyer A, et al. Autophagy in idiopathic pulmonary fibrosis. PLoS One 2012; 7:e41394.

50. Yamayoshi A, Oyama S, Kishimoto Y, et al. Development of Antibody-Oligonucleotide Complexes for Targeting Exosomal MicroRNA. Pharmaceutics 2020;12:545. 